\title{
The Dadongling Section - a Long High Resolution Record from the West of the Chinese Loess Plateau
}

The accompanying graphs (figure 4) present a distillation of the results discussed more fully in Chen et al. (1995).

\section{Graph A: Probe readings of magnetic susceptibility at $\mathbf{5} \mathbf{c m}$ intervals}

The proposed correlations between paleosols (represented by peaks in susceptibility) and interglacial Marine Isotope Stages (MIS) suggest that this part of the section spans at least 5 glacial cycles. The upper shaded areas denote the two parts of the profile for which detailed magnetic measurements and particle size analyses have been carried out on individual subsamples (see Graph B).

\section{Graph B: Two subsections of the magnetic measurements spanning parts of MIS 6 to $5 \mathrm{C}$ and 4 to 1 . The approximate age of the lower of the two analysed sections is 'pinned' by evidence for the paleomag- netic 'Blake Event' around 110kyr BP. It seems likely that part of the record from tation. Thus, the magnetic grains in the loess represent relatively coarse, wind- transported detrital material; the finer grains (three to four orders of magni- tude smaller volume) in the paleosols mainly represent the products of in-situ soil formation.} Stages $5 \mathrm{~A}$ and B is missing from the section and a hiatus is tentatively inferred just below $17 \mathrm{~m}$ depth. The early- to mid-Holocene age of the upper paleosol is confirmed by radiocarbon dates. The $5 \mathrm{~cm}$ sample interval used in the lower part of the section thus provides a temporal resolution of around $250 \mathrm{yr}$ or better; above this, there are intervals where suites of contiguous $1 \mathrm{~cm}$ thick samples provide a temporal resolution of around $50 \mathrm{yr}$. The record of variations in susceptibility $(\chi)$ outlines the changing concentration of magnetic minerals through the profiles. $\chi_{\mathrm{fd}} \%$ and $\chi_{\text {arm/SIRM }}$ provide mutually independent estimates of the proportion of magnetic minerals of pedogenic origin. The two sharp, pre-Holocene peaks in all three properties lie within the Late-Glacial period. The coherent and repeated submillennial scale oscillations within MIS 5C-D are considered further in the text below relating to Graph E.

Full references for this article can be found at www.pages.unibe.ch/ publications/newsletters/ref993.html

\section{Graph C: Magnetic properties measured on particle sized separates}

This figure provides a more robust basis for interpreting the magnetic contrast between the loess (Sample 1 - top of MIS 6) and paleosol (Sample 2 - MIS $5 \mathrm{E})$ parts of the record. Maximum magnetic concentrations are much lower in the late MIS 6 loess and peak in the coarse and medium silt fractions. Evidence for fine, pedogenic grains is virtually absent. Concentraions in the MIS 5E palaoesol sample peak in the finest clay fraction and pedogenic grains are dominant. The opposite trends in demagnetisation characteristics (reverse field ratios) versus particle size provide independent reinforcement for this interpre-

\section{Graph D: Magnetic property most} strongly indicative of pedogenesis $\left(\chi_{\mathrm{fd}} \%\right)$ against the mass percentage of particles coarser than $62.5 \mu \mathrm{m}$

Pedogenic episodes, the strength of which can be, at least qualitatively, differentiated by variations in $\chi_{\mathrm{fd}} \%$, show consistently low coarse silt percentages; conversely, the main episodes of loess deposition can be differentiated on the basis of the percentage coarse silt contribution, but $\chi_{\mathrm{fd}} \%$ remains consistently low. Less than $5 \%$ of the samples plotted contravene this general observation. This indicates that the two proxy signatures show a degree of independence that allows for credible magnetic-proxybased reconstructions of moisture and temperature regimes during interglacial periods dominated by the summer monsoon (cf. Maher and Thompson, 1999), and of changing wind strength, using particle size variations, during glacial periods dominated by the winter monsoon (cf. Chen et al. 1997). Both lines of inference have their limitations and require further critical evaluation, but the simple bi-plot helps to explain some of the ways in which loess profiles have been used to provide reconstructions of past climatic variations; for example, for the last glacial period, during which links between Heinrich layers in the North Atlantic and coarser granulometry in loess sections have been inferred.

\section{Graph E: high resolution record of mag- netic property variations during part of MIS 5C-D}

$\chi_{\text {arm }}$ may be used here as an indicator of the concentration of pedogenic grains, the properties on either side, as mutually independent indicators of their relative importance, as in Graph B. Over this time interval of around $15 \mathrm{kyr}$, there are at least 5 coherent alternations between periods of partial pedogenesis and intervals of loess deposition, each of the latter with little or no indication of soil development. These alternations occur on the same time scales as Dansgaard-Oeschger oscillations. These results suggest that (i) the continuity of eolian aggradation for key time intervals at sites of high accumulation in the western part of the Loess Plateau allows for an extremely high resolution reconstruction of short term climate changes, (ii) the magnetic signatures are essentially syndepositional in such environments and avoid the polygenetic overprinting more typical of paleosols in the classic loess sections further east, and (iii) putative links between North Atlantic variability and the stratigraphy of Chinese Loess sections on Dansgaard-Oeschger time scales are supported not only by granulometric data, but by magnetic measurements, though lack of a good chronology precludes direct correlations.

Frank Oldfield

PAGES IPO, Bern, Switzerland oldfield@pages.unibe.ch 


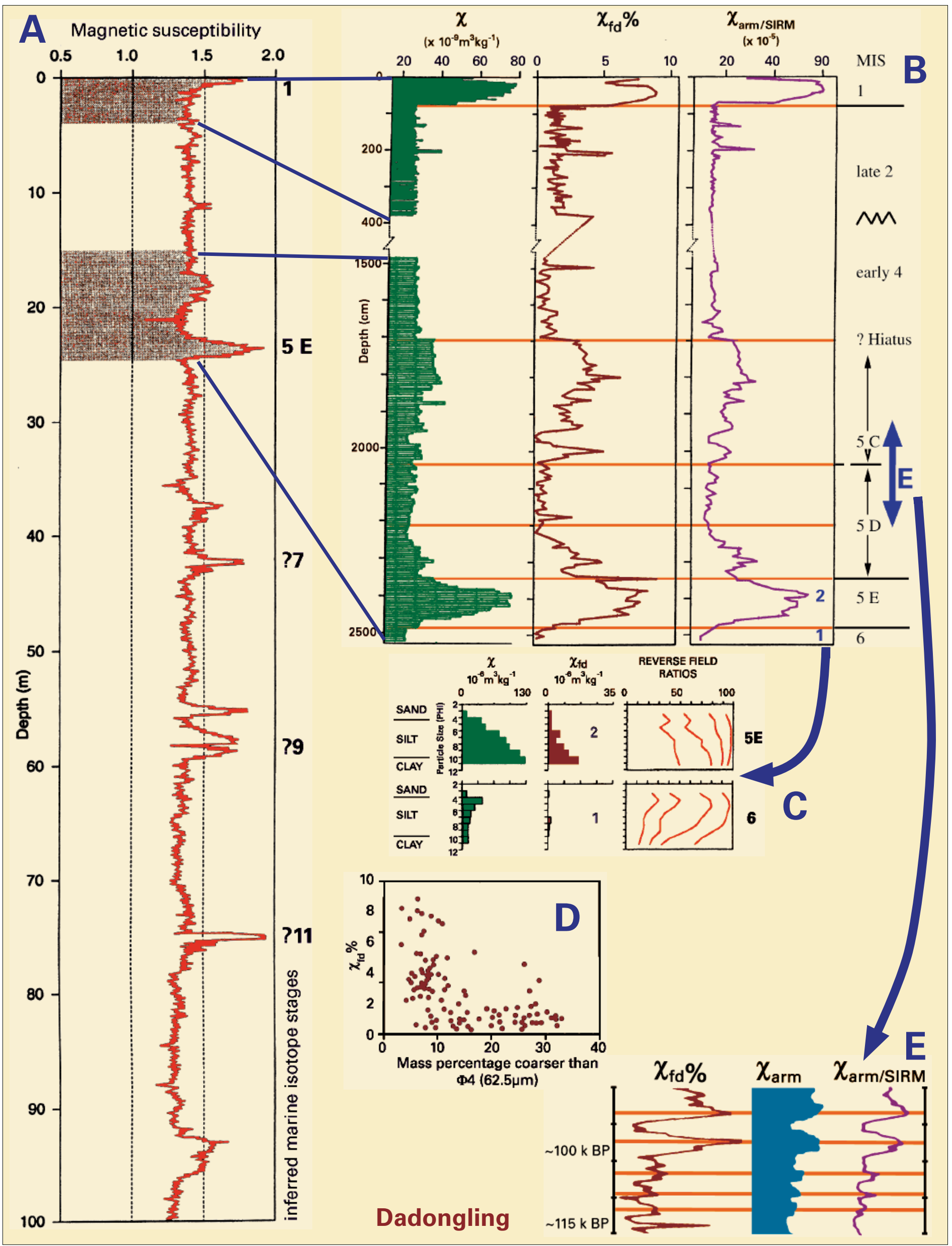

Figure 4: The Dadongling loess section 